\title{
A Case Report on Cilostazol Induced Loss of Diabetic Control
}

\author{
Dilip Chandrasekhar*, Nizar Mohamed Kanjhiraparambil, Drisya Rajan Chalappurath \\ Department of Pharmacy Practice, Al Shifa College of Pharmacy, Perinthalmanna, Kerala, INDIA.
}

\begin{abstract}
A 69-year-old male with known case of diabetes mellitus and hypertension was admitted with wet gangrene of right greater toe. He received cilostazol $100 \mathrm{mg}$ twice a day for 3 days. During this period, there was a marked elevation of blood sugar requiring higher dosage of insulin. Upon withholding the suspected drug there was a marked fall in blood glucose levels indicating a positive dechallenge. Cilostazol might have loss of diabetic control by favouring intestinal glucose absorption through increased cAMP levels and GLUT2 expression necessitating management with higher dosage of insulin.
\end{abstract}

Key words: Cilostazol, Wet gangrene, Hyperglycemia, Diabetes mellitus. Correspondence

Dr. Dilip Chandrasekhar, Department of Pharmacy, Practice Alshifa College of Pharmacy, Poonthavanam, Perinthalmanna, Kerala, INDIA.

Phone: +919447252670

Email: dillu7@gmail.com

DOI: 10.5530/jyp.2018.10.107

\section{INTRODUCTION}

Gangrene is a condition where there is a loss of blood supply leading to tissue necrosis. It affects every part of the body typically starting in the toes, feet, fingers and hands. Wet gangrene involves an infection which spreads throughout the body and form tissue swells and blisters. It usually occurs due to either the blockage of venous blood flow or due to blood vessel occlusion. Cilostazol is a drug that is indicated for reduction of symptoms of intermittent claudication and secondary prevention of cerebral infarction associated with gangrene. ${ }^{1}$

Cilostazol is an antiplatelet agent that belongs to the category of reversible selective inhibitor of phosphodiesterase type III which induces an increase in cyclic AMP in platelets, vascular smooth muscles and endothelial cells. It functions as an inhibitor of platelet aggregation with vasodilation effect. Cilostazol is indicated for reduction of symptoms of intermittent claudication. ${ }^{1}$ In peripheral vascular diseases, treatment with cilostazol $50-100 \mathrm{mg}$ bid is preferred. ${ }^{2}$

Drug-induced disturbances to glucose metabolism have either been due to idiosyncrasy or coincidence. ${ }^{3}$ According to FDA adverse event reporting system, there has been $0.27 \%$ incidence of increased blood glucose with cilostazol. ${ }^{4}$ We here in report a case of cilostazol induced loss of diabetic control in a 69 year old male patient.

\section{CASE REPORT}

A 69 year old male patient with known case of Diabetes Mellitus and Hypertension, on treatment with insulin, was presented with swelling and pain on right greater toe persisting for the last 5 days. Physical examination revealed Wet Gangrene on right greater toe. Investigations revealed an increase in total white blood cells, polymorphs and erythrocyte sedimentation rate and a reduction in lymphocytes.

The patient had a mean glycemic level of $232 \mathrm{mg} / \mathrm{dl}$ prior to the therapy with phosphodiesterase inhibitor Cilostazol (Figure 1). During treatment with cilostazol 100mg (b.i.d), the mean glucose levels increased from $232 \mathrm{mg} / \mathrm{dl}$ to $521 \mathrm{mg} / \mathrm{dl}$.

So cilostazol 100mg (b.i.d) was withheld. There was a significant reduction in the elevated blood glucose level to $172 \mathrm{mg} / \mathrm{dl}$. The elevated glucose levels were managed with Human Mixtard 40 Units. Intravenous Human Atrapid ( from 5 IU upto 30IU) was administered on the day following the administration of cilostazol. The patient was treated with Cefoperazone $1 \mathrm{~g}+$ sulbactam $1 \mathrm{~g}$ (b.i.d), metronidazole 500mg (b.i.d), pantoprazole $40 \mathrm{mg}$ (b.i.d), probiotics $5 \mathrm{mg}$ (b.i.d), tolvaptan $15 \mathrm{mg}$ once daily, sodium bicarbonate (b.i.d), calcitriol $0.2 \mathrm{mg}$ once daily, aspirin $150 \mathrm{mg}$ once daily, human actrapid 30 units-30 units- 16 units and human mixtard 0-0-40 units.

\section{DISCUSSION}

Recently conducted studies found cilostazol to ameliorate impairment of $\beta$-cell viability and insulin-secretory responses to glucose in patients with type 2 diabetes. ${ }^{1}$ Some studies have demonstrated stimulation of both glycogenolysis and gluconeogenesis in hepatocytes after administration of Phosphodiesterase inhibitors. ${ }^{1}$ Clinical studies evaluating the effects of cilostazol on glycemic control are scarce. ${ }^{1}$ Following the administration of Cilostazol, there was a marked rise in the blood glucose levels even after insulin administration. Though the clinical condition of diabetes can be conclusive of high blood glucose levels, the patient's

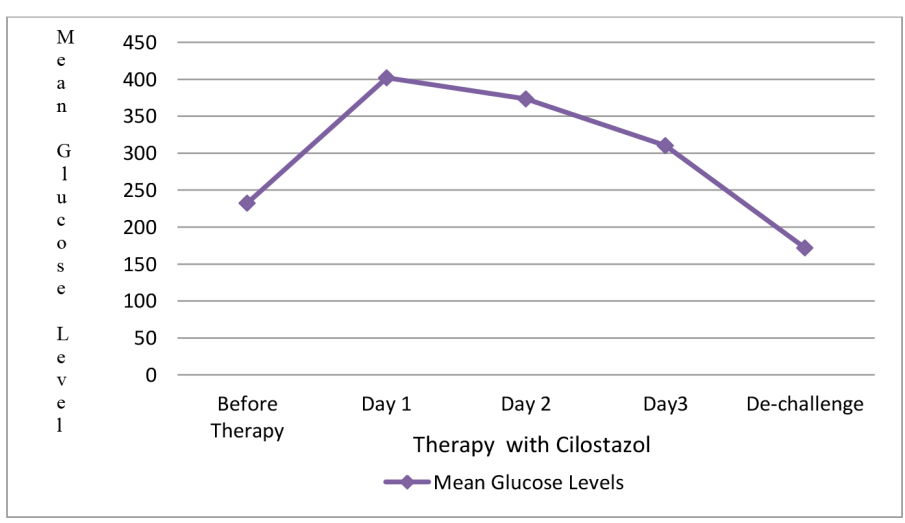

Figure 1: Comparison of mean glucose levels before the Cilostazol therapy, with therapy and after stopping the drug. 
insulin requirement continued to rise until cilostazole was withdrawn from administration.

The probable cause of hyperglycemia in the above case was hypothesised to be cilostazol induced as dechallenge with the drug produced a remarkable reduction in the elevated blood glucose levels. None of the concomitant medications were known to cause hyperglycemia.

According to the Naranjo Scale for causality assessment, the association of hyperglycemia with the use of cilostazol was probable with a score of 5. The mechanism of hyperglycemia with cilostazol can be explained with the significant elevated expression of GLUT 2 on jejunal erythrocytes. ${ }^{2}$ Cilostazol increases cAMP by phosphodiesterase inhibition. It clinically appears to have the potential to elevate the blood glucose level, presumably via the overproduction of glucose in the liver beyond the insulin-secretory capacity. ${ }^{1}$ It is known to favour intestinal glucose absorption through cAMP-involved direct pathway. ${ }^{2}$ Hence it was proposed that cilostazol could cause drug induced loss of diabetic conrolin this patient. The glucose metabolism may be affected by drugs in response to several other factors that include maintenance of balance between release and action of insulin, counter-regulatory response of glucagon, growth hormones, catecholamine and cortisol. ${ }^{3}$ Even though its effect on glucose metabolism is unclear, ${ }^{1}$ several host related factors like glucoregulatory mechanism, comorbidities, organ function and other concurrent medications may also contribute to risk of drug- induced hyperglycemia. ${ }^{3}$

\section{CONCLUSION}

This case report has revealed that cilostazol can be causally related to hyperglycemic event observed. Though the pharmacologic effect of cilostazol in vasodilation and inhibition of platelet aggregation is well known, possible effects of this drug on glycemic control should be assessed. The observed hyperglycemic effect of the drug can be turned into a therapeutic benefit in patients with refractory hypoglycaemia. ${ }^{3}$ Further assessment of possible effects of cilostazol in glycemic control must be paid much more attention along with consideration of risk- benefit ratio of cilostazol use and individual circumstances. ${ }^{1}$

\section{CONFLICT OF INTEREST}

The authors declare no conflict of interest.

\section{REFERENCES}

1. Yuji A, Mika S, Nobuaki W. The blood glucose level increased in parallel with the heart rate following cilostazol administration in three diabetic patients. Intern Med. 2014;53:859-63.

2. Kanaka DV, Britto DL, Shanmugapriya S, Saravanan T. Deterioration of glycemic control induced by pentoxifylline and cilostazol in a diabetic patient with peripheral vascular disease. IJOPP. 2013;6:69-71.

3. Juliana C N C, Clive S C, Julian A J H C. Drug-induced disorders of glucose metabolism. Drug Safety. 2012;15:135-57.

4. United states Food and Drug Administration's Adverse Event Reporting System [internet] 2012 [ updated 2012 ; cited 2004].

Article History: Submission Date : 01-06-2018; Revised Date : 03-07-2018; Acceptance Date : 27-07-2018.

Cite this article: Chandrasekhar D, Mohamed NKP, Rajan DC. A Case Report on Cilostazol Induced Loss of Diabetic Control. JYoung Pharm. 2018;10(4):489-90. 